\title{
Diverse Cavity Types and Evidence that Mechanical Action on the Necrotic Granuloma Drives Tuberculous Cavitation
}

\author{
Elizabeth A. Ihms, ${ }^{* \dagger}$ Michael E. Urbanowski, ${ }^{\dagger}$ and William R. Bishai
}

From the Department of Molecular and Comparative Pathobiology, ${ }^{*}$ and the Center for Tuberculosis Research, ${ }^{\dagger}$ Department of Medicine, Division of Infectious Diseases, Johns Hopkins University School of Medicine, Baltimore, Maryland

\author{
Accepted for publication \\ April 3, 2018. \\ Address correspondence to \\ William R. Bishai, M.D., Ph.D., \\ Center for Tuberculosis \\ Research, Department of Medi- \\ cine, Division of Infectious \\ Diseases, Johns Hopkins \\ School of Medicine, CRB2, \\ Room 108, 1550 Orleans St., \\ Baltimore, MD 21287. E-mail: \\ wbishai1@jhmi.edu.
}

\begin{abstract}
Effacement of normal lung parenchyma by cavities is an important sequela of pulmonary tuberculosis. Despite its clinical significance, the pathogenesis of tuberculous cavitation is poorly understood, with controversy as to whether the fundamental mechanism involves matrix depletion, lipid pneumonia, or mechanical factors. In this study, a repetitive aerosol infection model using Mycobacterium tuberculosis was used to generate cavities in $20 \mathrm{New}$ Zealand white rabbits. Serial computed tomography was performed to monitor cavity progression over 14 weeks. Three-dimensional reconstructions were compiled for each time point, allowing comprehensive four-dimensional cavity mapping. Terminally, cavities were processed for histopathology. Cavities progressed rapidly from areas of consolidation, and often showed a pattern of explosive growth followed by gradual contraction. Cavities formed preferentially in the caudodorsal lung fields, and frequently were subpleural. Cavitation was associated invariably with necrosis. Histomorphology showed four distinct cavity types that provide mechanistic clues and insight on early cavity development. Our study shows that cavitation is a highly dynamic process with preferential formation at sites of high mechanical stress. These findings suggest a model for the pathogenesis of tuberculous cavitation in which mechanical stress acts on the necrotic granuloma to produce acute tears in structurally weakened tissue, with subsequent air trapping and cavity expansion. (Am J Pathol 2018, 188: 1666-1675; https://doi.org/10.1016/j.ajpath.2018.04.006)
\end{abstract}

Despite the availability and efficacy of current antimycobacterial drugs, tuberculosis (TB) continues to be a significant cause of morbidity and mortality worldwide. To combat this persistent epidemic, research efforts have focused primarily on treatment and prevention with the development of new antibiotics and vaccines. However, the more difficult question of reducing transmission in highburden countries has been insufficiently addressed. Thirty percent to $50 \%$ of active TB infections result in the development of pulmonary cavities: abnormal air spaces surrounded by scar tissue that have replaced the normal lung parenchyma. ${ }^{1}$ Although not unique to TB, cavitation plays a central role in the epidemiology of tuberculosis by driving transmission, treatment failure, and drug resistance. ${ }^{1-4}$ The contribution of cavitation to the continued spread of TB makes it an attractive target for therapeutic intervention. However, despite the clear epidemiologic importance of cavitary disease, the pathophysiology of cavity development remains controversial.

The most widely accepted model of cavity formation proposes that cavities arise when large granulomas undergo central liquefaction, and subsequently erode into adjacent airways. Once in communication with the respiratory tree, these lesions discharge their liquefied contents, leaving behind an air-filled cavity. In this model, enzymatic proteolysis and matrix destruction are considered the major drivers of cavitation, and, indeed, multiple matrix remodeling enzymes are known to be up-regulated surrounding cavitary lesions. ${ }^{5-10}$ With this body of literature, matrix

Supported by NIH grants AI36973, AI37856, and HL133190 (W.R.B.); and by the Howard Hughes Medical Institute (W.R.B.).

E.A.I. and M.E.U. contributed equally to this work.

Disclosures: None declared. 
metalloproteinases (MMPs) have emerged as promising potential therapeutic targets. Although MMP inhibition in animal models clearly reduces extracellular matrix turnover, ${ }^{11}$ cavity formation has not been reduced by agents that block MMP activity in mice or rabbits (A.A. Ordonez, personal communication, March 2017). ${ }^{11,12}$ Extracellular matrix depletion is expected to generate significant erosions into bronchioles and larger airways, and thus predicts sustained communication with the bronchial tree. Such cavities should have a well-aerated interior, with a similar gaseous composition to that of inspired air. However, studies by Haapanen et $\mathrm{al}^{13}$ showed that the majority of human cavities have an interior gas composition that is hypoxic and hypercarbic relative to alveoli, and frequently display either positive or negative internal pressure. These findings agree with earlier work by Coryllos and Ornstein, ${ }^{14}$ who showed that cavities frequently contain pressurized interiors. More recently, investigations by Belton et $\mathrm{al}^{15}$ showed strong uptake of fluoromisonidazole labeled with fluorine-18-an indicator of severe tissue hypoxia-in the consolidated region immediately surrounding pulmonary cavities. These findings show that sustained bronchiolar communication is not reliably present in the majority of cavities.

A second model of tuberculous cavitation proposes that cavities arise within the context of lipid pneumonia, independent of the granuloma. Drivers of cavitation in this model include bronchial obstruction by tuberculous pneumonia, and mycobacterial elaboration of cord factor. ${ }^{16,17}$ Support for this model is based largely on human autopsy studies from the 19th and early 20th centuries. Consequently, it is difficult to link the identified lesions at autopsy with premortem serial three-dimensional radiologic imaging (eg, computed tomography [CT] scanning) and thereby establish a chronology of events leading to cavitation. Importantly, lipid pneumonia has not been linked with cavitation in animal models, the majority of which show that cavitation occurs within the context of the caseating granuloma. $^{7,9,18,19}$

Finally, the role of mechanical forces in the context of pulmonary cavitation appears as a persistently unanswered question over many decades of TB literature $\mathrm{e}^{20-25}$ and recently has received renewed attention. ${ }^{26,27}$ The mechanical model of cavitation has its origins in the preantibiotic era, and postulates that cavitation is the result of the highly dynamic environment of the lung, which is subjected to constant motion and fluctuating pressures not experienced elsewhere in the body. ${ }^{28}$ Pioneers of this model were the research surgeons of the preantibiotic era, who used iatrogenic pneumothorax to "rest" the lung and allow closure of the cavity. ${ }^{21, p .652}$ Although invasive, this frequently was successful. ${ }^{22}$ This model is supported by the observation that cavities in humans form preferentially in the apices of the lung, coinciding with the highest levels of pleural stress. ${ }^{28}$ Additional insight has been gained from comparisons with centrilobular emphysema and spontaneous pneumothorax, which have a similar preference for the lung apex. ${ }^{25,26}$ Similar to the lipid pneumonia model, the mechanical model emphasizes the role of bronchial obstruction with formation of one-way valves that allow air to enter a cavity but prevent its escape. ${ }^{14}$ Despite pulmonary mechanics being cited frequently as a probable contributor to cavitation, few investigators have attempted to clarify the role of mechanical stress in the pathogenesis of tuberculosis, likely owing to limitations of current model systems.

A major challenge in investigating cavitary disease is that, of the available animal systems used to model TB, few develop spontaneous cavitary disease that shows phenotypic similarity to the human counterpart. ${ }^{18,19}$ Our laboratory has developed a reliable model of tuberculous cavitation using outbred New Zealand white rabbits infected with a serial low-dose exposure of Mycobacterium tuberculosis. This infection protocol was designed to mimic the pattern of human exposure in endemic environments. A high percentage of animals develop cavities by 7 to 9 weeks after infection, and cavities display a high degree of phenotypic similarity to human cavitary lesions (Supplemental Figure S1). ${ }^{29}$ By using high-resolution CT in combination with gross pathology and histopathology, we were able to follow cavities in real time and gain insight into the dynamics of cavity formation. By using this infection protocol in conjunction with high-resolution CT scans, we show that mechanical factors play a significant role in cavity generation and maintenance.

\section{Materials and Methods}

\section{Mycobacterial Culture}

Cultures of $M$ tuberculosis $\mathrm{H} 37 \mathrm{Rv}$ were grown from frozen stock aliquots in Middlebrook 7H9 liquid medium (Becton Dickinson, Sparks, MD) supplemented with $0.5 \%$ glycerol and $0.5 \%$ Tween 80 (Sigma-Aldrich, Darmstadt Germany).

\section{Animal Infection}

Twenty SPF adult female New Zealand white Rabbits, weighing 3.5 to $4.0 \mathrm{~kg}$ (Charles River Laboratories, Wilmington, MA, and Robinson Services, Mocksville, NC) were singly housed under Biosafety level 3 conditions in accordance with protocols approved by the Institutional Animal Care and Use Committee at Johns Hopkins University (Baltimore, MD). Five low-dose aerosol exposures over a 2.5-week period using a Madison aerosol infection chamber were used for infecting (College of Engineering, University of Wisconsin-Madison, Madison, WI). Delivery inoculum averaged $10^{6}$ organisms per chamber cycle (six animals per cycle), and animals were randomized between inoculations to minimize intercycle variability. This resulted in an implantation of approximately 500 colony-forming units per animal per exposure. Colony-forming unit determination was calculated as previously described. ${ }^{12}$ Sensitization was confirmed in all animals 5 days after the final 
inoculation by intradermal injection of $0.1 \mathrm{~mL}$ mammalian old tuberculin in the right flank (Zoetis, Parsippany, NJ). Induration was read at 48 hours, and was quantified by calculating the average skin fold thickness in two dimensions multiplied by the ratio of the thickness at affected to unaffected sites. All animals were visually inspected daily for signs of illness, including respiratory distress, or a reduction in appetite, urinary, or fecal output. Animals were weighed biweekly, at which time they received brief physical examinations by a licensed veterinarian.

\section{Thoracic CT}

Animals were sedated with intramuscular injections of xylazine $(3 \mathrm{mg} / \mathrm{kg})$, ketamine $(25 \mathrm{mg} / \mathrm{kg})$, and acepromazine $(1 \mathrm{mg})$ before bronchoscopically assisted intubation with a $3.5-\mathrm{mm}$ endotracheal tube (Teleflex Medical, Research Triangle Park, $\mathrm{NC})$. After intubation, anesthetized animals were placed into a sealed chamber for transport to an imaging suite, where they were maintained on injectable anesthesia and 1 to $3 \mathrm{~L} /$ minute oxygen. Breath-hold image acquisition was performed as previously described. ${ }^{30}$ Sedation was partially reversed with i.v. yohimbine $(0.1 \mathrm{mg} / \mathrm{kg})$ as needed. All animals were imaged at baseline (before infection), and then at 5, 7, 9, 11 to 12 , and 14 weeks after infection on a CereTom portable CT scanner (NeuroLogica, Boston MA).

\section{Image Processing}

Raw CT scan data were compiled into DICOM files using AMIDE software version 0.9.0. ${ }^{31}$ All image analysis was performed using VivoQuant software version 2.50 (Invicro, Boston, MA). Cavities were defined as having a central radiodensity matching that of aerated lung (-1024 to -725 Hounsfield units), surrounded by a radiodensity matching that of soft tissue ( $>-725$ Hounsfield units), which had a spherical morphology in three dimensions. Cavities that were not identified on at least two consecutive time points (including necropsy) were excluded from analysis to minimize the inclusion of radiographic artifacts. Gross photographs were analyzed in ImageJ software version 1.49 (NIH, Bethesda, MD; http://imagej.nih.gov/ij) by manually segmenting diseased lung and calculating the extent of disease as the ratio of involved to uninvolved lung in approximately 5 -mm transverse lung sections.

\section{Necropsy and Histopathology}

I.M. sedation was performed as described in Thoracic $C T$, after which animals were euthanized with 2 to $3 \mathrm{~mL}$ i.v. pentobarbital solution in the marginal ear vein. Immediately after euthanasia, the lungs were isolated and gently infused with $10 \%$ neutral buffered formalin before fixation. Tissues were fixed in $10 \%$ neutral buffered formalin for 48 hours, at which time they were serially sectioned, photographed, and processed for histology. Five-micron paraffin-embedded sections were stained with hematoxylin and eosin, or Masson's trichrome. Image capture for semiautomated quantification of trichrome staining was performed on a Nikon Eclipse 90i microscope with an attached Nikon DS-Ri1 color camera, and analyzed using NIS Elements Advanced Research software version 4.50 (Nikon Instruments, Melville, NY). Region of interest selection was performed manually, and included the entire thickness of the cavity wall while excluding the interior air space. Positive staining then was calculated as a percentage of the total region of interest.

\section{Statistical Analysis}

All statistical analysis was performed with GraphPad Prism software version 7.0 (GraphPad Software, San Diego, CA). Single-variable data were analyzed by the nonparametric Kolmogorov-Smirnov test to compare cumulative distributions. Paired data were analyzed by the nonparametric Wilcoxon test. Data sets containing three or more groups were analyzed by one-way analysis of variance with the Kruskal-Wallis test. The degree of correlation was calculated by linear regression with Spearman correlation coefficients and 95\% CI. All error bars represent means and SD.

\section{Results}

\section{Cavities Show Rapid Expansion Compared with Solid Caseous Lesions}

Cavitation was observed beginning at week 7 after infection, with the majority of cavities arising between 9 and 14 weeks after infection. High interanimal variability was observed in terms of disease severity and cavity generation, with $65 \%$ (13 of 20) of animals developing at least one cavity, and $69 \%$ (9 of 13) of those developing more than one cavity. Cavity size varied considerably, both within and between animals (Supplemental Table S1).

Cavitation invariably was preceded by a region of dense consolidation, identified as pulmonary regions with radiographic density matching that of nonaerated soft tissue $(>-725$ Hounsfield units) (Figure 1, A and B). To examine how cavities transition from these areas of solid consolidation, total lesion volume $\left(\mathrm{V}_{\text {consolidation }}+\mathrm{V}_{\text {cavity }}\right)$ between sequential CT time points was compared with $\mathrm{V} 2$ representing the first appearance of the cavitary lesion, and V1 representing the region of consolidation at the time point immediately preceding cavitation. The V2: V1 ratio then was used to assess the rate of growth for each lesion. Cavity growth was rapid and abrupt, with cavity volumes ranging from 0.67 to 9.30 times the volume of the regions of consolidation from which they arose. On average, cavities were 1.87 times larger than their respective consolidated foci. This then was compared with the growth rates of solid, noncavitary lesions over the same time frame (sequential time points between weeks 7 and 14) using 


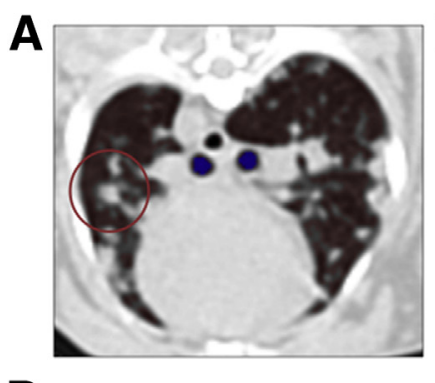

D

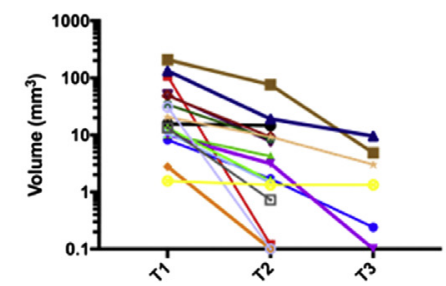

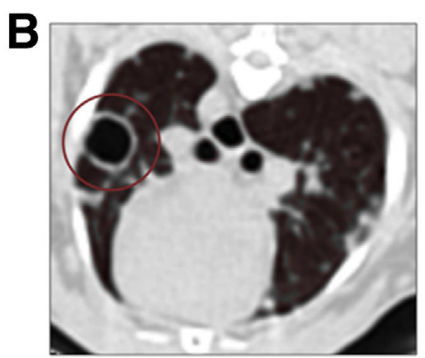

Growing

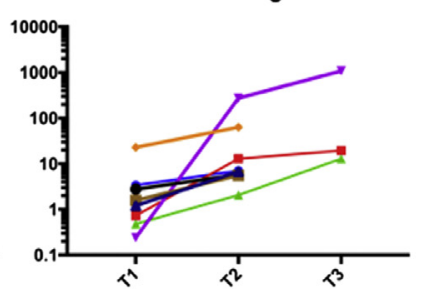

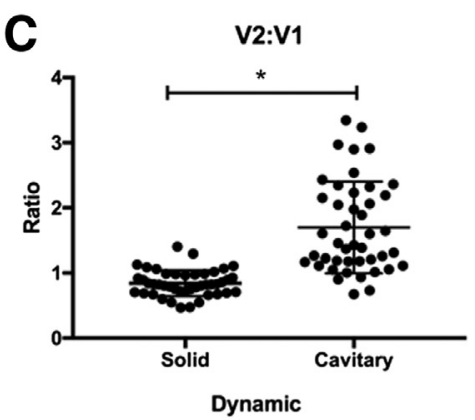

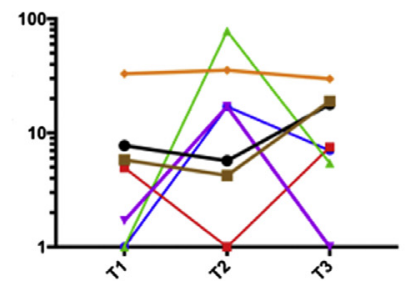

Figure 1 Cavities arise rapidly from areas of pulmonary consolidation, and show highly dynamic growth kinetics. A: Midthoracic transverse computed tomography (CT) of an infected rabbit at week 7 after infection shows a focus of pulmonary consolidation $4.4 \mathrm{~mm}$ in diameter, with no evidence of cavitation (red circle). B: Midthoracic transverse CT of the same animal at week 9, showing a large air-filled cavity present in the area of previous consolidation, measuring $8.8 \mathrm{~mm}$ in diameter (red circle). C: Growth rates of solid and cavitary lesions during week 7 to 14 after infection, where V1 and V2 represent the lesion volume at two sequential time points. D: Growth dynamics of cavitary lesions during week 7 to 14 after infection, where T1 represents the first appearance of the cavity, and T2 and T3 represent sequential time points after the cavitation event. Each colored line represents a single cavity. Data are expressed as means $\pm \mathrm{SD}(\mathbf{C}) .{ }^{*} P<0.0001$, Kolmogorov-Smirnov paired $t$-test.

the method specified earlier in this paragraph. The behavior of solid lesions was significantly less dynamic, with growth rates ranging from 0.47 to 1.57 . Surprisingly, $78 \%$ of noncavitary lesions examined had a V2:V1 ratio $<1$, indicating that the majority of solid lesions shrunk over this time period (Figure 1C).

After formation, cavity behavior was highly variable, with cavities falling into one of three groups. The majority of cavities (17 of 32) were largest at the time of initial detection, and then progressively contracted over subsequent time points. A second group of cavities (8 of 32) underwent gradual enlargement from the time of detection. This group included several of the largest cavities. Finally, a third group ( 7 of 32) was characterized by small size $(<100$ $\mathrm{mm}^{3}$ ) and dynamic behavior with alternating growth and shrinkage (Figure 1D). Four percent of cavities (3 of 79) underwent complete resolution, becoming undetectable on both radiography and gross pathology. Remarkably, resolution did not correspond to any specific growth pattern. Of the three cavities that resolved completely, one fell into each of the groups described earlier in this paragraph: growing, shrinking, and dynamic.

Disease Is Distributed Evenly throughout the Lung, but Cavitation Occurs at Regions of High Mechanical Stress

Because cavitary disease in human patients affects the apices of the lungs disproportionally, this study examined whether cavities in rabbits also displayed a bias toward specific lung regions. Although there was no significant difference in the distribution of solid lesions in the lungs
(Supplemental Figure S2A), pulmonary cavities were distributed unevenly throughout the lung, with significantly more cavities forming in the caudal lobes (Figure 2A). To determine the effects of transpulmonary pressures on cavity formation, a plane was drawn on CT images parallel to the course of the primary bronchi at the level of the tracheal bifurcation. This separated the lung into cranioventral and caudodorsal lung fields, corresponding to the sites of lowest and highest transpulmonary pressure in quadrupeds, respectively. ${ }^{32}$ Significantly more cavities formed in the caudodorsal lung fields when compared with the cranioventral regions, on both a total and per-animal basis, with animals having roughly twice as many caudodorsal cavities on average (Figure 2B). Cavities in the caudodorsal fields also were larger than those in the cranioventral regions, and were associated with significantly more collagen deposition (Figure 2, C and D). Irrespective of lung lobe, cavities usually (33 of 49) displayed a subpleural localization, in which they were associated with varying degrees of pleural inflammation and fibrosis. Surprisingly, despite frequent proximity to the pleural space, there was no evidence of cavitary rupture or pneumothorax, although occasional fibrinous adhesions between the visceral and parietal pleura were noted in several animals on postmortem examination.

\section{Cavitation Occurs Exclusively in the Context of Necrotizing Granulomas with Variable Airway Communication}

Histologically, $100 \%$ (49 of 49) of examined cavities occurred within the context of organized granulomas, 

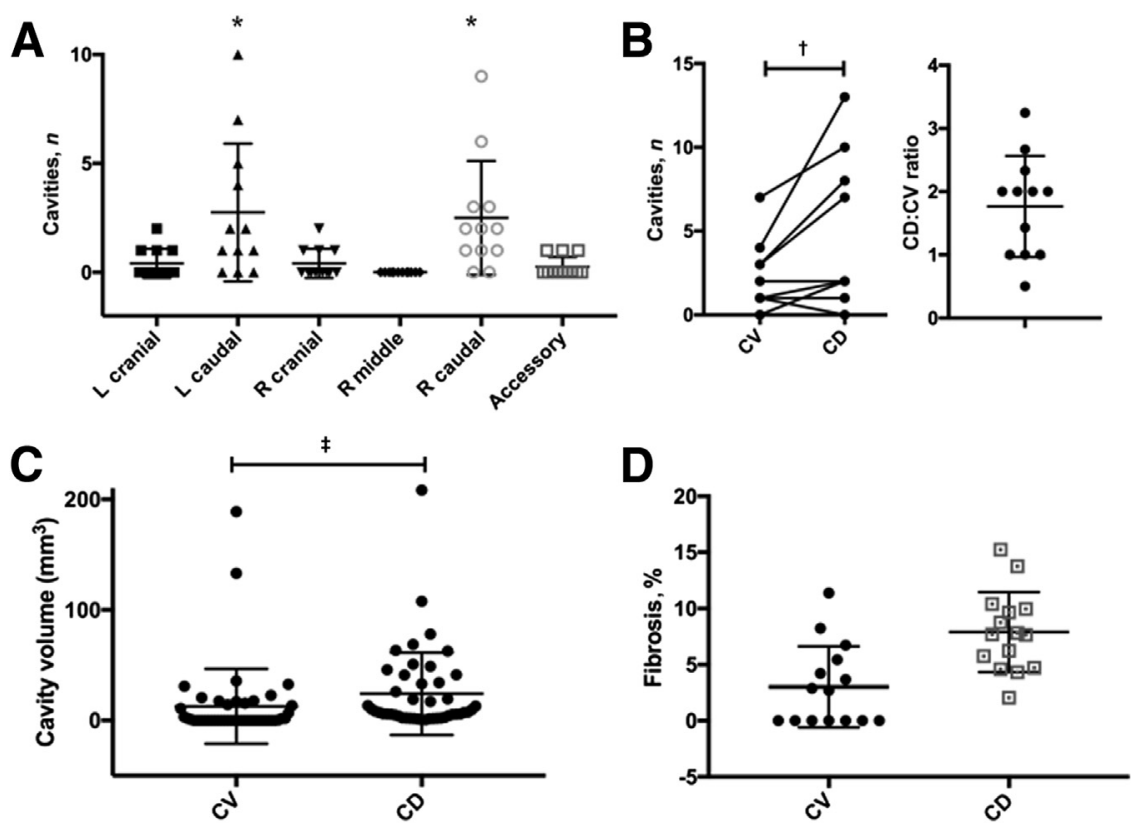

Figure 2 Cavities occur at regions of high mechanical stress, where they become larger and more fibrotic. A: Distribution of cavities within the lung of tuberculosis (TB)-infected rabbits, enumerated by computed tomography (CT) scan. Each point represents data from a single animal. Significantly more cavities form in the right and left caudal lobes. B: Cavities preferentially form in the caudodorsal (CD) lung fields when compared with cranioventral (CV) lung fields. Connected lines represent cavities in the same animal. C: Maximum volumes attained by cavities occurring in the CV or CD lung fields. D: Percentage of the cavity wall composed of fibrotic tissue, calculated by positive trichrome staining (collagen). Data are expressed as means \pm SD. ${ }^{*} P<0.0001$, one-way analysis of variance with the Kruskal-Wallis test; ${ }^{\dagger} P<0.05$, Wilcoxon paired $t$-test; ${ }^{\ddagger} P<0.0001$, Kolmogorov-Smirnov test; $P=0.0025$, Kolmogorov-Smirnov test (D). L, left; R, right. composed of discrete layers of epithelioid macrophages, multinucleated giant cells, and lymphocytes, surrounded by fibroblasts and partially encapsulated in mature collagen. Cavitating granulomas were associated universally with central necrosis, as evidenced by a variably thick rim of acellular granular debris lining the cavity interior. Organized granulomatous inflammation with central necrosis was a feature of all cavities examined, regardless of lesion age, size, morphology, or location within the lung. Although cavitation was associated invariably with necrosis, the converse was not true. Large necrotic granulomas without evidence of central cavitation were visualized frequently, suggesting that necrosis is necessary but not sufficient for the development of cavities (Supplemental Figure S1C). Airway communication, defined as histologic continuity of the cavity interior with ciliated columnar respiratory epithelium, was detected in only $16 \%$ ( 8 of 49 ) of examined cavities. These microscopic connections were below the limit of radiographic detection, and were not identified on CT. As such, this figure likely underestimates the prevalence of bronchiolar communications. Based on gas and pressure analyses performed by Haapanen et al, ${ }^{13}$ roughly $33 \%$ of cavities are predicted to have sustained open communication with an airway.

Our observations regarding rabbit tuberculous cavities were as follows: i) they expand abruptly, ii) they appear subpleurally in the caudodorsal lung fields that experience the highest transpulmonary pressure, and iii) they form exclusively from pre-existing necrotic granulomas consistent with cavity biogenesis resulting from acute tears in areas of necrotic tissue, with rupture into small airways and rapid subsequent gaseous filling.
Cavities Show Divergent Histomorphology with Four Discrete Lesion Types that Provide Mechanistic Clues

Histologic examination of cavities showed four distinct lesion morphologies (Figure 3A). The dominant morphology-designated as smooth-was characterized by a sharply defined gas tissue interface at the cavity interior (Figure 3B). This morphology was seen in more than half of the cavities examined (27 of 49). The least common morphology (rough; 4 of 49) had an interior cavity surface with an ill-defined, ragged, gas-tissue interface (Figure 3C). A third type (mixed; 13 of 49) showed characteristics of both rough and smooth cavities, with only a portion of the interior having the crisp and well-demarcated gas-tissue interface that defined smooth cavities (Figure 3D). The commonality of these three types was the relative thickness of the cavity wall. Smooth, rough, and mixed cavities contained variable amounts of necrotic debris at the interior surface, and were surrounded by a thick wall of organized granulomatous inflammation. Finally, a small subset of cavities (5 of 49), designated as fibrous, were characterized by large size and extremely thin walls composed almost entirely of mature fibrous tissue, and a slender discontinuous inner rim of necrotic and inflammatory debris (Figure 3E).

Next, the relationship between lesion morphology and lesion age, defined as the time from first CT appearance of the cavity to the time of cavity resolution or necropsy, was examined. Unexpectedly, cavities with smooth or fibrous morphology represented the youngest lesions (Figure 3F). A potential confounder in this analysis is the fact that $4 \%$ (3 of 79) of cavities had resolved by the time of necropsy, making it impossible to define their cavity types. Thus, it is possible 
A

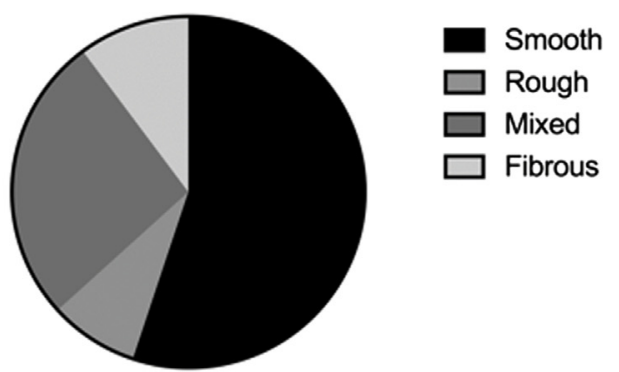

C

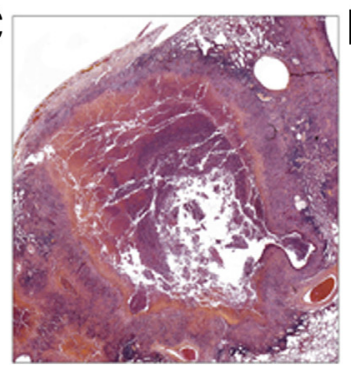

B
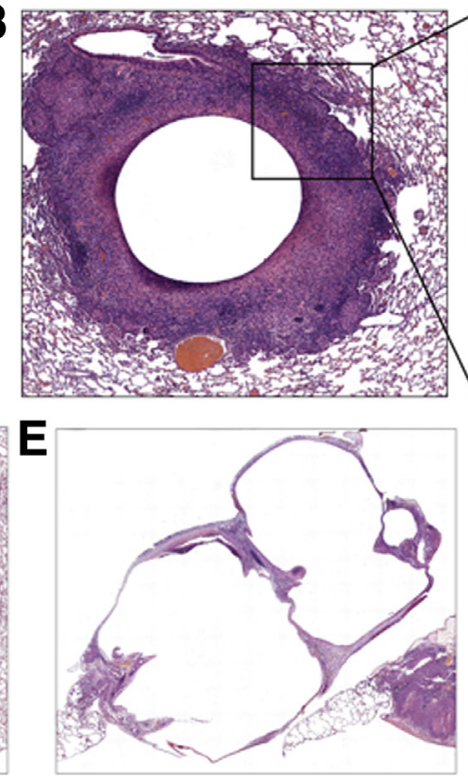

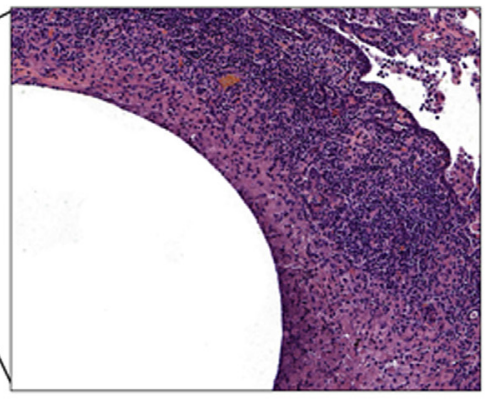

$\mathbf{F}$

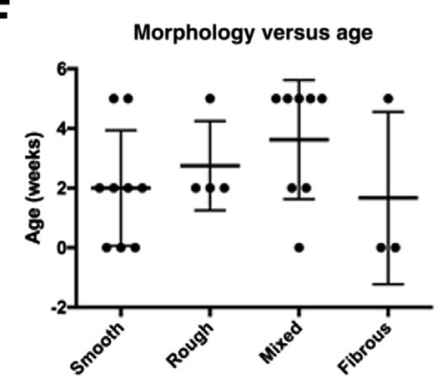

Figure 3 Histopathology shows divergent cavitary morphology. A: Histopathologic analysis of 49 cavitary lesions from 20 animals shows four distinct cavitary morphologies. B-E: Hematoxylin and eosin stains. B: A smooth pulmonary cavity with a sharply defined gas-tissue interface, adjacent to a bronchiole. Boxed area in left panel shows a higher magnification of interior surface of cavity in the right panel. C: A large subpleural cavity with rough morphology, associated with focal pleuritis and pleural fibrosis. D: A subpleural mixed cavity. The upper portion of the cavity has a smooth well-demarcated gas-tissue interface, whereas the lower portion is rough and ragged. The cavity has partially collapsed, leaving a dense band of fibrosis at the left aspect of the cavity. E: A very large fibrous or tension-type cavity expands and obliterates the tip of a lung lobe, extending to both pleural surfaces. The wall is composed of mature fibrous connective tissue lined by scant necrotic debris and minimal cellular infiltrate. F: Age of cavities based on serial CT scans and correlation with terminal histopathology. Data are expressed as means \pm SD (F).

that spontaneously resolving cavities belonged to a particular cavity type that would be under-represented in the analysis discussed in the previous paragraph.

All cavities were stained with Masson's trichrome to assess the amount of collagen in each lesion as a percentage of the total cavity wall, which excluded air and necrotic debris at the cavity interior. Not surprisingly, fibrous cavities displayed the highest amount of collagen as a total percentage of their wall, but there was no significant difference in collagen content among the other three cavity types (Supplemental Figure S2B). Remarkably, the amount of fibrosis did not correlate with the age of the lesions (Supplemental Figure S2C).

\section{Tuberculin Reactivity and Disease Severity Do Not Predict the Extent of Cavitation}

Previous work by Converse et $\mathrm{al}^{33}$ and Kubler ${ }^{30}$ showed a weak correlation between the degree of tuberculin reactivity and the extent of cavitation using a small number of animals. In the present study, no significant correlation was found between induration and either the severity of disease or the number of cavities generated (Figure 4, A and B). Somewhat unexpectedly, the extent of grossly visible disease also was not predictive of cavitation, and animals with minimal disease occasionally generated large numbers of cavities (Figure 4C).

\section{Discussion}

By using a longitudinal imaging approach in combination with histopathology, it was shown that cavities expand rapidly upon development and then may resolve, continue to grow, or wax and wane in size. Far from being a new observation, the dynamic nature of cavities appears as a common source of frustration as far back as the early 1900s, with Coryllos anthropomorphically relating their behavior as "capricious and puzzling." 22,p.301 When comparing the growth kinetics of cavities with solid lesions, it is apparent that there are mechanisms at work other than simple evacuation of interior contents. One would expect cavities growing by simple liquefaction and evacuation to be similar in size to the solid lesions that precede them. However, most cavities in this study were several times larger than their corresponding precursor lesions, suggesting an explosive growth event. In addition, the observation that most cavities are largest at the time of initial presentation strongly suggests that the onset of cavitation is an acute event, rather than a slow emptying of contents. The surprising observation that the amount of lesion fibrosis does not correlate with the age of the lesion confirms that remodeling of the fibrotic wall is similarly dynamic.

In humans, cavitary lesions show a strong preference for the apices of the lung. ${ }^{34}$ Based on this distribution, it has long been presumed that cavities form preferentially at sites of high ventilation:perfusion ratios, which favor bacterial 

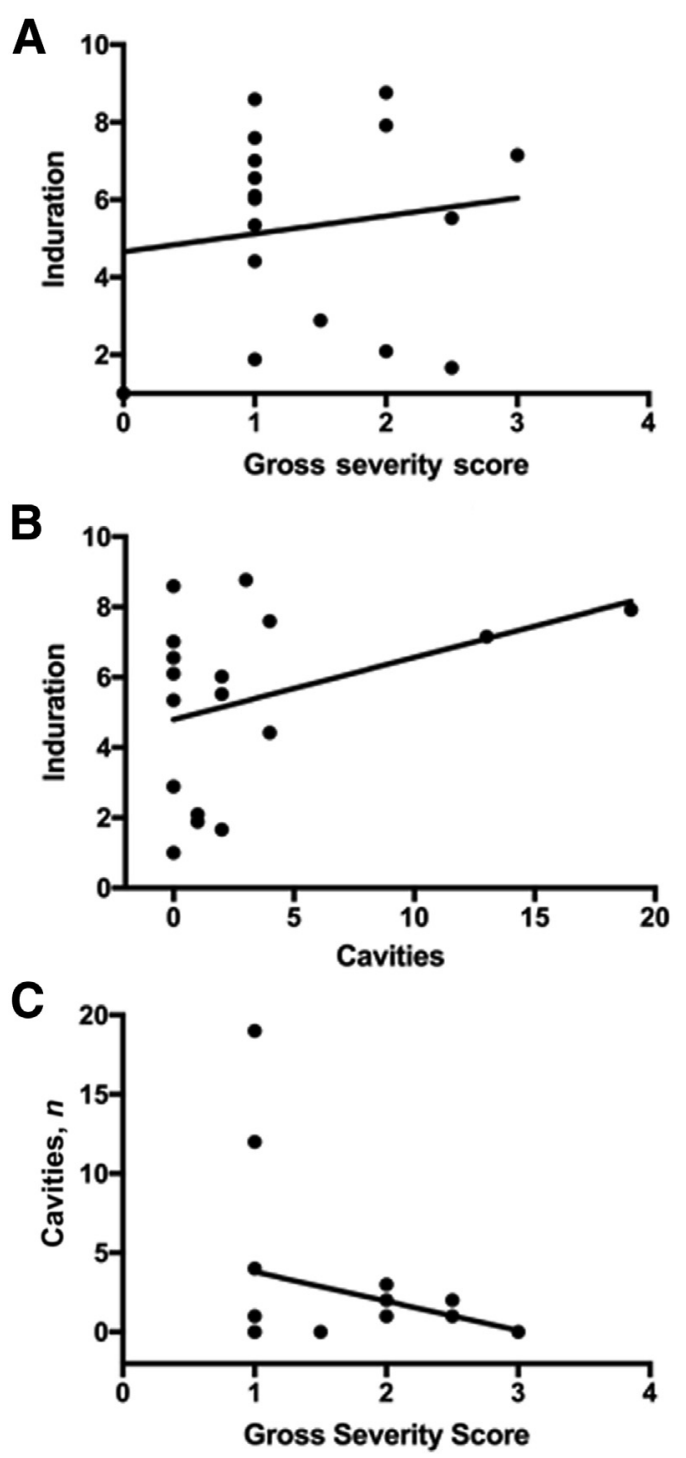

Figure 4 Tuberculin reactivity is not predictive of cavitation or disease severity. A: Scatter plot of the degree of induration at 48 hours after dermal old tuberculin and gross severity scores based on the percentage of lung affected. Each point represents an individual animal. $R^{2}=0.06$. B: Degree of induration at 48 hours plotted against the number of cavities. $R^{2}=0.13$. C: Number of cavitary lesions plotted against gross severity scores. Note animals with extensive cavitation but minimal gross pathology. $R^{2}=0.02$. Linear regression with Spearman correlation coefficients and $95 \%$ CI showing the line of best fit (solid black lines, all plots).

growth. ${ }^{27}$ However, in addition to receiving the highest relative ventilation, the apices of the human lung are also the site of highest mechanical stress. Transpulmonary pressure (TPP), the tension force applied to the lung by negative pressure within the pleural space, follows a vertical gradient owing to the interactions of gravity and thoracic wall shape. In humans, the area of greatest TPP therefore corresponds to the apices of the lung, ${ }^{25,28}$ whereas in quadrupeds, the caudodorsal lung fields experience the highest TPP. ${ }^{32}$ Meanwhile, ventilation:perfusion ratios in animals are distributed more uniformly throughout the lung as a result of the effects of body posture on blood flow. ${ }^{35,36}$ This critical species difference has allowed us for the first time to tease apart the relative contributions of ventilation:perfusion and TPP to the cavitation process.

Our observation that rabbit tuberculous cavities are twofold more likely to form in the caudodorsal lung fields in the face of uniform disease indicates that TPP gradients play a significant role in determining cavity distribution. In addition, these cavities are also larger, and display significantly increased collagen deposition when compared with those at low TPP sites. This is likely owing to the wellknown effects of biomechanical instability on collagen deposition in damaged tissue. ${ }^{37,38}$ These results expand on the findings of Medlar and Sasano, ${ }^{39}$ who reported that maintaining infected rabbits in an upright posture alters the distribution of their disease to a more human phenotype, with localization at the apices. Recent investigations by Casha et $\mathrm{al}^{26}$ further emphasize the role of mechanical stress in human cavity development. By using computer modeling techniques, they showed the profound effect that thoracic wall shape has on pleural stress, especially at the apex of the lung. In low body mass index chests, they discovered up to a 10 -fold increase in pleural stress at the apex, potentially explaining the increased incidence of cavitary TB in young males and individuals with low body mass index.

A majority of cavities in this study occurred subjacent to the pleura, further supporting the hypothesis that cavities occur at regions of structural weakness. Lesions occurring here are not surrounded by a buffer zone of elastic alveoli, but instead face the pleura, which contains a higher proportion of inelastic collagen. In the face of high regional TPP, this area is predisposed to tears, evidenced by the high frequency of subpleural blebs and bullae in other pulmonary diseases such as emphysema. ${ }^{25,26}$ Studies of patients with pleural tuberculosis (arising from extension of subpleural lesions) report the incidence of subpleural cavitation at 50\% to $60 \%$, but the fraction of cavities that form subpleurally is unclear. ${ }^{40}$ Cavitation is not unique to $\mathrm{TB}$, and may be caused by a diverse group of infectious etiologies as well as several neoplasms. The fraction of subpleural cavities has been documented for several other diseases, and in some infections approaches $100 \%$. $^{1}$

Histologic examination of a large number of lesions has shown the existence of distinct cavity types. More than half of cavities displayed a sharply demarcated gas - tissue interface, identical to those observed in the emergent $\mathrm{C} 3 \mathrm{HeB} / \mathrm{FeJ}$ mouse model of cavitation. ${ }^{9}$ Similar morphology has been referenced in detailed pathologic descriptions by Sweany and Seiler, who described cavities with "smooth walls,"29,p.140 and provided photomicrographs of human cavities with clear mixed and fibrous morphology (Supplemental Figure S1, D and E), confirming the translatability of findings in the rabbit model. This smooth histologic appearance is not pathognomonic but is distinctive of lesions in which gas exerts pressure on surrounding tissue. Other diseases that share this morphology include Clostridial gas gangrene, ${ }^{41}$ 
decompression sickness, ${ }^{42}$ and gas bubble disease in aquatic species, ${ }^{43}$ in which the production or traumatic inoculation of gas bubbles produces the appearance of compressed tissue surrounding a central air space. The gross appearance of large gas bubbles dissecting through tissue planes ${ }^{42}$ brings to mind Sweany and Seiler's descriptions of tuberculous cavities with "smooth and shining walls," 29, p.140 and also undeniably resembles the smooth shiny cavitary interior seen frequently in this model (Supplemental Figure S1A).

In the context of tuberculosis, these smooth-type cavities suggest a novel mechanism for cavity formation (Figure 5). The necrotic granuloma is fundamentally a sphere with a rigid fibrotic exterior and a soft caseous interior. Inspiration produces a rapid decrease in pleural pressure, translating to the application of external tension and development of negative pressure within the sphere. Fibrous tissue is inelastic, and therefore prone to failure under repetitive mechanical stress. A tear occurring in the fibrotic wall that extended to the necrotic center would allow air to be sucked into the soft interior on inspiration, with rapid subsequent enlargement of the lesion. Upon expiration, the tension force would be released, resulting in positive pressure within the lesion and temporary closure of the tear. This is similar to the one-way valve theory postulated by Coryllos and Ornstein, ${ }^{14}$ Coryllos, ${ }^{22}$ and Eloesser, ${ }^{23}$ and explains the finding that cavities frequently contain pressurized interiors. Cavities with smooth morphology are younger on average, confirming that this most likely represents an early stage of cavitation. An extreme example of air-trapping also may explain the presence of similarly young fibrous-type cavities, with their large central space and thin walls reminiscent of balloons. Experiments by Coryllos ${ }^{14}$ in 1938 corroborate this mechanism by the introduction of a smalldiameter thoracoscope into human cavities and injection of small amounts of dye or saline. When suction was applied to the cavity interior, Coryllos observed a "bubbling of air"14,p.15 inward through the bronchial outlet, with simultaneous drainage of the instilled fluid. However, when positive pressure was applied, no movement of air or saline occurred, indicating that the outlet had been obstructed temporarily. ${ }^{14}$

Our findings strongly support a mechanism of cavitation driven by mechanical action on the necrotic granuloma. However, the question of the role of matrix remodeling enzymes in cavitary pathogenesis remains. In addition to driving a number of pathologic conditions, MMPs play an essential physiological role in wound repair. ${ }^{44}$ Tissue injury by any means produces a secondary increase in matrix remodeling enzymes, and these increases persist in the case of chronic nonhealing wounds. Thus, increased levels of

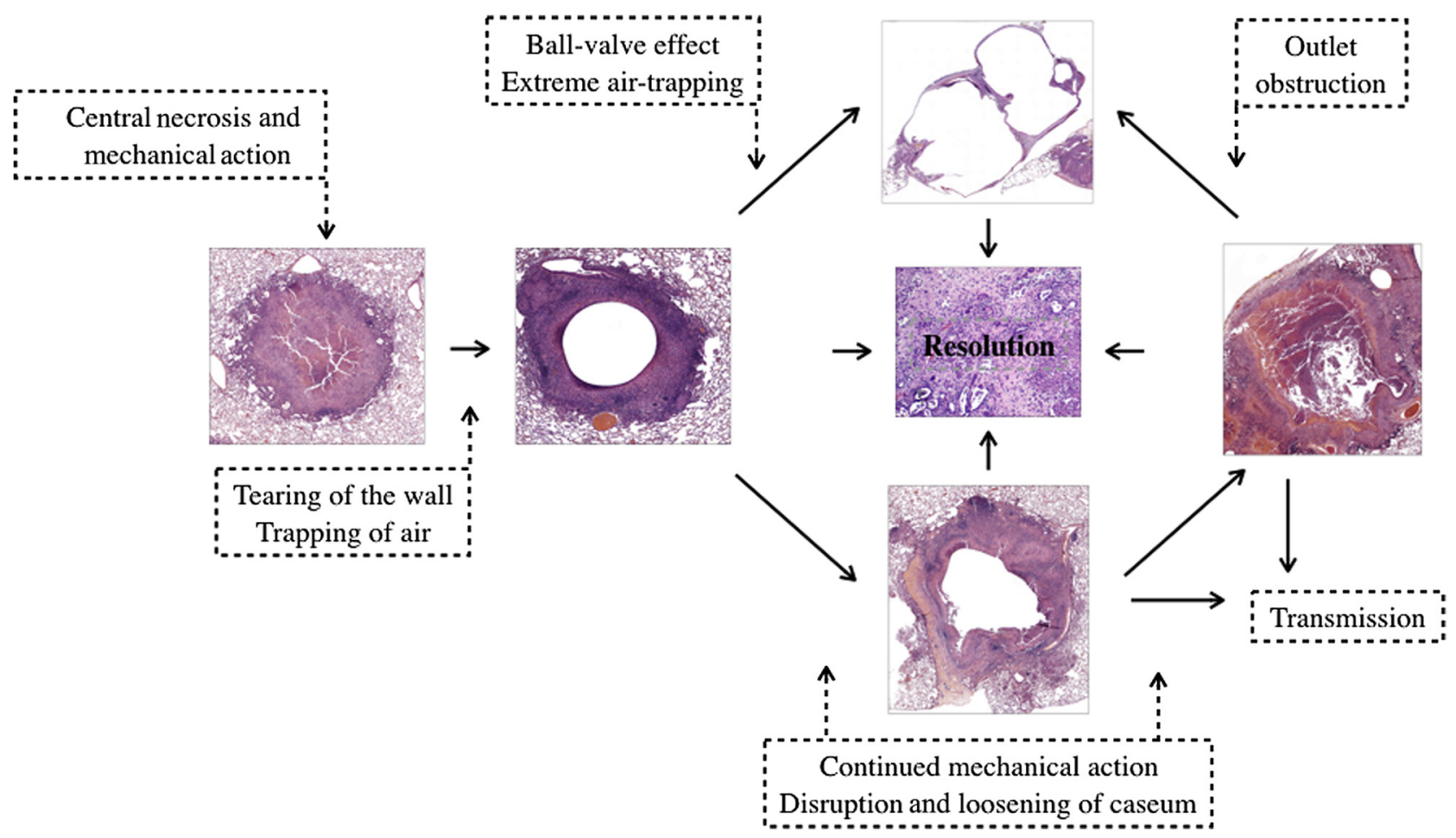

Figure 5 Proposed mechanism of cavity genesis and progression. A cavity begins when mechanical action disrupts the necrotic granuloma by causing a tear in the fibrotic wall. With inspiration, external tension in the surrounding lung tissue pulls air into the necrotic center, causing a smooth cavity. If at any point the outlet is severely obstructed creating a ball-valve effect, extreme air trapping occurs and causes the cavity to assume a thin-walled fibrous balloonlike morphology. If the outlet is open or only partially obstructed, air accumulates less rapidly, resulting in smaller cavities that may progress or wax and wane. The mechanical actions of respiration continue to act on these lesions, causing eventual disruption and loosening of the retained caseum. This newly freed material is coughed up in the sputum to continue the transmission cycle, leaving behind a mixed or rough cavity with a wall of progressively crumbling caseum. Finally, any of these lesions may spontaneously close and resolve, leaving a residual scar. 
MMPs and other extracellular matrix-modifying enzymes may reflect their action as secondary responders to tissue injury, rather than primary drivers of cavitation. Regardless of the initiating cause, it is likely that proteolysis and mechanical forces ultimately act in concert to produce tears in structurally weakened tissue.

Investigative pathology necessitates a snapshot approach that often makes determining a sequence of events challenging, if not impossible. For this reason, precursor lesions were documented radiographically for each cavity, and used to determine growth kinetics. There has been extensive discussion of liquefaction of the caseum as the critical event preceding cavitation by Canetti, ${ }^{34}$ Dannenberg, ${ }^{45}$ and Cardona and many others, ${ }^{27}$ which has induced a decades-long search for the missing link in cavity pathogenesis. The lack of grossly liquefied caseum in this model (in contrast to high-dose bronchoscopic and Mycobacterium bovis infection models) combined with the higher incidence of observed cavitation ${ }^{12}$ argues against the existence of a transitional lesion requiring liquefied caseum. Our data suggest that a cavity is merely a traumatized necrotic granuloma, and predict that the lesion that gives rise to a cavity is histologically indistinguishable from any other caseating granuloma. This is supported by the observation that residual caseum within a cavity is microscopically identical to caseum inside a solid necrotic granuloma in both humans and rabbits ${ }^{19}$ (Supplemental Figure S1).

In summary, this study used a rabbit model of cavitation that produced a high frequency of human-like cavities, serial high-resolution CT scanning, and careful histopathologic analysis to chronicle the kinetics, distribution, and histologic types of tuberculous cavities. These data support a mechanism of cavitation in which mechanical intrapulmonary forces act on the necrotic granuloma to produce a cavity. Although more studies are needed to confirm the findings here and their translatability to human patients, elucidation of these mechanical factors may provide additional insight on the lung-specific nature of cavitation. We also show further support that necrosis is an absolute prerequisite for cavitation. Because intrapulmonary mechanical action cannot be altered easily, future studies should endeavor to prevent necrosis as a means of inhibiting cavitation.

\section{Acknowledgments}

We thank Joseph Mankowski and Joshua Croteaux for microscopy support and software training, Mariah Klunk for imaging and technical support, and Pat Wilcox for histopathology laboratory support.

\section{Supplemental Data}

Supplemental material for this article can be found at https://doi.org/10.1016/j.ajpath.2018.04.006.

\section{References}

1. Gadkowski LB, Stout JE: Cavitary pulmonary disease. Clin Microbiol Rev 2008, 21:305-333

2. Perrin FMR, Woodward N, Phillips PPJ, McHugh TD, Nunn AJ, Lipman MCI, Gillespie SH: Radiological cavitation, sputum mycobacterial load and treatment response in pulmonary tuberculosis. Int J Tuberc Lung Dis 2010, 14:1596-1602

3. Benator D, Bhattacharya M, Bozeman L, Burman W, Catanzaro A, Chaisson R, Gordin F, Horsburgh CR, Horton J, Khan A, Lahart C, Metchock B, Pachucki C, Stanton L, Vernon A, Villarino ME, Yong CW, Weiner M, Weis S, Horsburgh CR, Burman W: Rifapentine and isoniazid once a week versus rifampicin and isoniazid twice a week for treatment of drug-susceptible pulmonary tuberculosis in HIVnegative patients: a randomised clinical trial. Lancet 2002, 360: $528-534$

4. Gillespie SH: Evolution of drug resistance in Mycobacterium tuberculosis: clinical and molecular perspective. Antimicrob Agents Chemother 2002, 46:267-274

5. Elkington P, Shiomi $\mathrm{T}$, Breen R, Nuttall RK, Ugarte-Gil CA, Walker NF, Saraiva L, Pedersen B, Mauri F, Lipman M, Edwards DR, Robertson BD, D'Armiento J, Friedland JS: MMP-1 drives immunopathology in human tuberculosis and transgenic mice. J Clin Invest 2011, 121:1827-1833

6. Elkington PT, D'Armiento JM, Friedland JS: Tuberculosis immunopathology: the neglected role of extracellular matrix destruction. Sci Transl Med 2011, 3:71ps6

7. Kübler A, Luna B, Larsson C, Ammerman NC, Andrade BB, Orandle M, Bock KW, Xu Z, Bagci U, Molura DJ, Marshall J, Burns J, Winglee K, Ahidjo BA, Cheung LS, Klunk M, Jain SK, Kumar NP, Babu S, Sher A, Friedland JS, Elkington PT, Bishai WR: Mycobacterium tuberculosis dysregulates MMP/TIMP balance to drive rapid cavitation and unrestrained bacterial proliferation. J Pathol 2015, 235: 431-444

8. Kubler A, Larsson C, Luna B, Andrade BB, Amaral EP, Urbanowski M, Orandle M, Bock K, Ammerman NC, Cheung LS, Winglee K, Halushka M, Park JK, Sher A, Friedland JS, Elkington PT, Bishai WR: Cathepsin K contributes to cavitation and collagen turnover in pulmonary tuberculosis. J Infect Dis 2016, 213:618-627

9. Ordonez AA, Tasneen R, Pokkali S, Xu Z, Converse PJ, Klunk MH, Mollura DJ, Nuermberger EL, Jain SK: Mouse model of pulmonary cavitary tuberculosis and expression of matrix metalloproteinase-9. Dis Model Mech 2016, 9:779-788

10. Walker NF, Wilkinson KA, Meintjes G, Tezera LB, Goliath R, Peyper JM, Tadokera R, Opondo C, Coussens AK, Wilkinson RJ, Friedland JS, Elkington PT: Matrix degradation in human immunodeficiency virus type 1-associated tuberculosis and tuberculosis immune reconstitution inflammatory syndrome: a prospective observational study. Clin Infect Dis 2017, 86:913-922

11. Walker NF, Clark SO, Oni T, Andreu N, Tezera L, Singh S, Saraiva L, Pedersen B, Kelly DL, Tree JA, D'Armiento JM, Meintjes G, Mauri FA, Williams A, Wilkinson RJ, Friedland JS, Elkington PT: Doxycycline and HIV infection suppress tuberculosis-induced matrix metalloproteinases. Am J Respir Crit Care Med 2012, 185:989-997

12. Urbanowski ME, Ihms EA, Bigelow K, Kubler A, Elkington PT, Bishai WR: Repetitive aerosol exposure promotes cavitary tuberculosis and enables screening for targeted inhibitors of extensive lung destruction. J Infect Dis 2018. [Epub ahead of print] doi: 10.1093/infdis/jiy127

13. Haapanen JH, Kass I, Gensini G, Middlebrook G: Studies on the gaseous content of tuberculous cavities. Am Rev Respir Dis 1959, 80: $1-5$

14. Coryllos PN, Ornstein GG: Giant tuberculous cavities of the lung. J Thorac Surg 1938, 8:10-54

15. Belton M, Brilha S, Manavaki R, Mauri F, Nijran K, Hong YT, Patel NH, Dembek M, Tezera L, Green J, Moores R, Aigbirhio F, 
Al-Nahhas A, Fryer TD, Elkington PT, Friedland JS: Hypoxia and tissue destruction in pulmonary TB. Thorax 2016, 71: $1145-1153$

16. Hunter RL, Olsen MR, Jagannath C, Actor JK: Multiple roles of cord factor in the pathogenesis of primary, secondary, and cavitary tuberculosis, including a revised description of the pathology of secondary disease. Ann Clin Lab Sci 2006, 36:371-386

17. Hunter RL: On the pathogenesis of post primary tuberculosis: the role of bronchial obstruction in the pathogenesis of cavities. Tuberculosis 2011, 91:S6-S10

18. Helke KL, Mankowski JL, Manabe YC: Animal models of cavitation in pulmonary tuberculosis. Tuberculosis 2006, 86:337-348

19. Leong FJ, Dartois V, Dick T: A Color Atlas of Comparative Pathology of Pulmonary Tuberculosis. Boca Raton, FL, CRC Press, 2010

20. Gekler W, Lovelace W, Rankin H, Weigel B: Tuberculous cavitation of the lung: mechanical factors in its genesis, and combined chemotherapeutic and surgical treatment. JAMA 1924, 82:457-463

21. Coryllos PN: The mechanics and biology of tuberculous cavities. Am Rev Tuberc 1936, 33:639-660

22. Coryllos PN: Physics applied to tuberculosis. J Franklin Inst 1939, 227:287-304

23. Eloesser L: Blocked cavities in pulmonary tuberculosis. J Thorac Surg 1937, 7:1-22

24. Medlar EM: The behavior of pulmonary tuberculous lesions; a pathological study. Am Rev Tuberc 1955, 71:1-244

25. West JB: Distribution of mechanical stress in the lung, a possible factor in localization of pulmonary disease. Lancet 1971, 297:839-841

26. Casha AR, Camilleri L, Manché A, Gatt R, Attard D, Wolak W, Dudek K, Gauci M, Giordimaina C, Grima JN: A hypothesis for reactivation of pulmonary tuberculosis: how thoracic wall shape affects the epidemiology of tuberculosis. Clin Anat 2015, 28:614-620

27. Cardona P-J: A spotlight on liquefaction: evidence from clinical settings and experimental models in tuberculosis. Clin Dev Immunol 2011, 2011:868246

28. Agostoni E: Mechanics of the pleural space. Physiol Rev 1972, 52: $57-128$

29. Sweany HC, Seiler HH: The pathology and bacteriology of resected lesions in pulmonary tuberculosis. Dis Chest 1956, 29:119-152

30. Kubler A: Development and Investigation of a Rabbit Model of Tuberculosis Tissue Destruction. London, UK: Imperial College London, 2013

31. Loening AM, Gambhir SS: AMIDE: a free software tool for multimodality medical image analysis. Mol Imaging 2003, 2(3):131-137
32. D’Angelo E, Bonanni MV, Michelini S, Agostoni E: Topography of the pleural surface pressure in rabbits and dogs. Respir Physiol 1970, 8:204-229

33. Converse PJ, Dannenberg AM, Shigenaga T, Mcmurray DN, Phalen SW, Stanford JL, Rook GAW, Koru-Sengul T, Abbey H, Estep JE, Pitt AMLM: Pulmonary bovine-type tuberculosis in rabbits: bacillary virulence, inhaled dose effects, tuberculin sensitivity, and mycobacterium vaccae immunotherapy. Clin Diagn Lab Immunol 1998, 5:871-881

34. Canetti G: Thé Tubercle Bacillus in the Pulmonary Lesion of Man. New York, Springer International Publishing, 1955

35. Wagner PD, Laravuso RB, Umm RR, Wesm JB: Continuous distributions of ventilation-perfusion ratios in normal subjects breathing air and $100 \% \mathrm{O}_{2}$. J Clin Invest 1974, 54:54-68

36. Yokoyama T, Farhi LE: Study of ventilation-perfusion ratio distribution in the anesthetized dog by multiple inert gas washout. Respir Physiol 1967, 3:166-176

37. Suki B, Ito S, Stamenović D, Lutchen KR, Ingenito EP: Biomechanics of the lung parenchyma: critical roles of collagen and mechanical forces. J Appl Physiol 2005, 98:1892-1899

38. Wells RG: Tissue mechanics and fibrosis. Biochim Biophys Acta 2013, 1832:884-890

39. Medlar E, Sasano K: A study of pathology of experimental tuberculosis. Am Rev Tuberc 1936, 34:456

40. Hulnick DH, Naidich DP, McCauley DI: Pleural tuberculosis evaluated by computed tomography. Radiol 1983, 149:759-765

41. Tsokos M, Schalinski S, Paulsen F, Sperhake JP, Püschel K, Sobottka I: Pathology of fatal traumatic and nontraumatic clostridial gas gangrene: a histopathological, immunohistochemical, and ultrastructural study of six autopsy cases. Int J Legal Med 2008, 122:35-41

42. Jepson PD, Arbelo M, Deaville R, Patterson IAP, Castro P, Baker JR, Degollada E, Ross HM, Herráez P, Pocknell AM, Rodríguez F, Howie FE, Espinosa A, Reid RJ, Jaber JR, Martin V, Cunningham AA, Fernández A: Gas-bubble lesions in stranded cetaceans. Nature 2003, 425:575-576

43. Speare DJ: Histopathology and ultrastructure of ocular lesions associated with gas bubble disease in salmonids. J Comp Pathol 1990, 103: 421-432

44. Gill SE, Parks WC: Metalloproteinases and their inhibitors: regulators of wound healing. Int J Biochem Cell Biol 2008, 40:1334-1347

45. Dannenberg AM: Liquefaction and cavity formation in pulmonary TB: a simple method in rabbit skin to test inhibitors. Tuberculosis 2009, 89: 243-247 